\title{
THE HAUSDORFF DIMENSION OF SYSTEMS OF SIMULTANEOUSLY SMALL LINEAR FORMS
}

\section{H. DICKINSON}

Introduction. In this paper the Hausdorff dimension of systems of real linear forms which are simultaneously small for infinitely many integer vectors is determined. A system of real linear forms,

$$
\sum_{i=1}^{m} a_{i} x_{i j}, \quad j=1,2, \ldots, n,
$$

where $a_{i}, x_{i j} \in \mathbb{R}, 1 \leqslant i \leqslant m, 1 \leqslant j \leqslant n$ will be denoted more concisely as

$$
\mathbf{a} X \text {, }
$$

where $\mathbf{a} \in \mathbb{R}^{m}, X \in \mathbb{R}^{m n}$ and $\mathbb{R}^{m n}$ is identified with $M_{m \times n}(\mathbb{R})$, the set of real $m \times n$ matrices. The supremum norm $|\mathbf{v}|_{\infty}=\max \left\{\left|v_{1}\right|, \ldots,\left|v_{k}\right|\right\}$ of any vector in $k$ dimensional Euclidean space, $\mathbb{R}^{k}$ will be denoted by $|\mathbf{v}|$. The distance of a point $a$ from a set $B$, will be denoted by dist $(a, B)=\inf \{|a-b|: b \in B\}$.

Let $\tau>0$. The set of points (matrices) $X$ in $\mathbb{R}^{m n}$ such that

$$
|\mathbf{q} X|<|\mathbf{q}|^{-\tau}
$$

for infinitely many $\mathbf{q} \in Z^{m}$ will be denoted by $W(m, n ; \tau)$. For $m \geqslant 2$ the Hausdorff dimension of the set $W(m, n ; \tau)$ will be shown to be $(m-1) n+(m /(\tau+1))$ if $\tau>(m / n)-1$, and $m n$ if $\tau \leqslant(m / n)-1$. (For the definition of Hausdorff dimension see [6].) The case $n=1$ was solved in [5] but the methods used there cannot be used in the more general case dealt with here.

Let $I$ be the interval $\left(-\frac{1}{2}, \frac{1}{2}\right)$. To prove the lower estimate we shall use instead of $W(m, n ; \tau)$ its subset

$$
\hat{W}(m, n ; \tau)=\left\{X \in I^{m n}:|\mathbf{q} X|<|\mathbf{q}|^{-\tau} \text { for infinitely many } \mathbf{q} \in \mathbb{Z}^{m}\right\}
$$

in the working of the lemmas. Note that this set can be expressed in the general "lim sup" form

$$
\hat{W}(m, n ; \tau)=\bigcap_{N=1}^{\infty} \bigcup_{q>N} \bigcup_{\substack{|\mathbf{q}|=q \\ \mathbf{q} \in \mathbb{Z}^{m}}}\left\{X \in I^{m n}:|\mathbf{q} X|<|\mathbf{q}|^{-\tau}\right\} .
$$

It is readily verified that the set $W(1, n ; \tau)=\{0\}$ and so obviously the dimension in this case is 0 . Thus, from now on, unless otherwise stated, only the case $m \geqslant 2$ is considered. 
THEOREM 1. When $\tau>(m / n)-1$ and $m \geqslant 2$

$$
\operatorname{dim} W(m, n ; \tau)=(m-1) n+\frac{m}{\tau+1},
$$

and when $0<\tau \leqslant(m / n)-1$

$$
\operatorname{dim} W(m, n ; \tau)=m n
$$

The first part of the theorem is proved by finding upper and lower bounds for the Hausdorff dimension separately. The calculations for the upper inequality are simple but they are included for completeness. The lemma is true for any cube but is proved for $I^{m n}$ for convenience.

LEMMA 1. For any positive integers $m, n \geqslant 1$

$$
\operatorname{dim} \hat{W}(m, n ; \tau) \leqslant\left\{\begin{array}{lll}
(m-1) n+(m /(\tau+1)) & \text { for } & \tau>(m / n)-1, \\
m n & \text { for } & 0<\tau \leqslant(m / n)-1
\end{array}\right.
$$

Proof. As the points $X$ are contained in $\mathbb{R}^{m n}$, the Hausdorff dimension of $\hat{W}(m, n ; \tau)$ is at most $m n$.

Denote by $\Pi(\mathbf{q})$ the set $\left\{X \in I^{m n}: \mathbf{q} X=\mathbf{0}\right\}$. Now let $t>(m-1) n+(m /(\tau+1))$ and $\tau>(m / n)-1$. For each non-zero $q \in \mathbb{Z}^{m}$, the number of $m n$-dimensional hypercubes $C$ of width $L(C)=4|\mathbf{q}|^{-(\tau+1)}$ with centres on the $(m-1) n$ dimensional hyperplane $\Pi(\mathbf{q})$ at integral multiples of $|\mathbf{q}|^{-(\tau+1)}$ apart, with sides parallel to the axes, is

$$
\ll|\mathbf{q}|^{(\tau+1)(m-1) n},
$$

where $\ll f(x)$ means $\leqslant c f(x)$ for some positive constant $c$. The collection $\mathscr{C}(\mathbf{q})$ of such hypercubes $C$ covers

$$
B(\mathbf{q})=\left\{X \in I^{m n}:|\mathbf{q} X|<|\mathbf{q}|^{-\tau}\right\}
$$

and for each $N=1,2, \ldots$, the collection

$$
\mathscr{C}_{N}=\{\mathscr{C}(\mathbf{q}):|\mathbf{q}|>N\}
$$

covers $\hat{W}(m, n ; \tau)$.

The $t$-volume of $\mathscr{C}_{N}$ is defined by

$$
L^{t}\left(\mathscr{C}_{N}\right)=\sum_{C \in \mathscr{C}_{N}} L(C)^{t}=\sum_{\substack{\mathbf{q} \in \mathbb{Z}^{m} \\|\mathbf{q}|>N}} \sum_{C \in \mathscr{G}(\mathbf{q})} 4^{t}|\mathbf{q}|^{-(\tau+1) t},
$$

and, for $N$ sufficiently large,

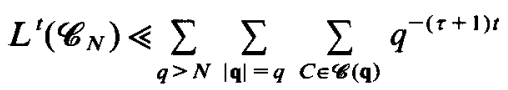

$$
\begin{aligned}
& \ll \sum_{q>N} q^{-(\tau+1) t+(m-1)+(\tau+1)(m-1) n}<\varepsilon,
\end{aligned}
$$

since $t>(m-1) n+(m /(\tau+1))$. Hence $\inf _{N \rightarrow \infty} L^{t}\left(\mathscr{C}_{N}\right)=0$ and the lemma follows from the definition of Hausdorff dimension.

Note that this bound is not best possible when $m=1$. 
The lower bound is obtained in two parts. Firstly the idea of ubiquity, introduced in [4], is used to find a bound in the case $m>n$. In the complementary case where $m \leqslant n$, a diffeomorph of $W(m, n ; \tau)$ is decomposed into a cartesian product of two spaces, one of which is an $(m-1)(n-m+1)$ dimensional cube. The arguments for the case $m>n$ apply to the other space. The following definition of ubiquity for affine spaces is easily shown to be equivalent to that in [4], where further details are given. Note that the Lebesgue measure of a set $A$ is written $|A|$.

Ubiquity. Let $\Omega$ be a non-empty bounded open region in $\mathbb{R}^{m n}$ and let

$$
\Pi(\mathbf{q})=\{X \in \Omega: \mathbf{q} X=\mathbf{0}\} .
$$

Denote the family of non-empty subsets of $\Omega$

$$
\left\{\Pi(\mathbf{q}): \mathbf{q} \in \mathbb{Z}^{m} \backslash\{\mathbf{0}\}\right\}
$$

by $\mathscr{R}$. Also for each $\delta>0$ write

$$
B(\Pi(\mathbf{q}) ; \delta)=\{X \in \Omega: \operatorname{dist}(X, \Pi(\mathbf{q}))<\delta\} .
$$

Let

$$
A(N)=\bigcup_{|\mathbf{q}| \leqslant N} B(\Pi(\mathbf{q}) ; \rho(N)) .
$$

Then, if $\lim _{N \rightarrow \infty}|\Omega \backslash A(N)|=0$ and $\lim _{N \rightarrow \infty} \rho(N)=0$ the family $\mathscr{R}$ is called a ubiquitous system relative to $\rho$. (For the details see [4].)

Now assume that $m>n$ and take $\Omega$ as

$$
\Omega=\left\{X \in I^{m n}: X \text { is of maximal rank } n\right\},
$$

i.e., the column vectors $\mathbf{x}^{(j)} \in I^{m}, 1 \leqslant j \leqslant n(\leqslant m-1)$ of the matrix $X$ are linearly independent. Note that $\Omega$ is an open subset of $I^{m n}$. Denote the set

$$
\left\{X \in \Omega:|\mathbf{q} X|<|\mathbf{q}|^{-\tau} \text { for infinitely many } \mathbf{q} \in \mathbb{Z}^{m}\right\}
$$

by $\tilde{W}(m, n ; \tau)$. Let $\psi(|\mathbf{q}|)=m^{-1}|\mathbf{q}|^{-(\tau+1)}$. Then, since $\Omega$ is a subset of $I^{m n}$,

$$
\hat{W}(m, n ; \tau) \supseteq \tilde{W}(m, n ; \tau) .
$$

Also,

$$
\tilde{W}(m, n ; \tau) \supseteq \Lambda
$$

where

$$
\Lambda=\left\{X \in \Omega: \operatorname{dist}(X, \Pi(\mathbf{q}))<\psi(|\mathbf{q}|)<\psi(|\mathbf{q}|) \text { for infinitely many } \mathbf{q} \in \mathbb{Z}^{m}\right\} .
$$

To see this let $X \in \Lambda$ and for non-zero $\mathbf{q} \in \mathbb{Z}^{m}$, let $U=\left(\mathbf{u}^{(1)}, \ldots, \mathbf{u}^{(n)}\right) \in \Pi(\mathbf{q})$ be the nearest point to $X$ so that $|X-U|<\psi(|\mathbf{q}|)$. Then, for each column vector $\mathbf{x}^{(j)}$ of $X \in \Lambda$,

$$
\begin{aligned}
\left|\mathbf{q} \cdot \mathbf{x}^{(j)}\right| & =\left|\mathbf{q} \cdot \mathbf{x}^{(j)}-\mathbf{q} \cdot \mathbf{u}^{(j)}+\mathbf{q} \cdot \mathbf{u}^{(j)}\right|=\left|\mathbf{q} \cdot \mathbf{x}^{(j)}-\mathbf{q} \cdot \mathbf{u}^{(j)}\right| \\
& =\left|\mathbf{q} \cdot\left(\mathbf{x}^{(j)}-\mathbf{u}^{(j)}\right)\right| \leqslant|\mathbf{q}|_{2}\left|\mathbf{x}^{(j)}-\mathbf{u}^{(j)}\right|_{2} \\
& \leqslant m^{1 / 2}|\mathbf{q}|_{\infty} m^{1 / 2}\left|\mathbf{x}^{(j)}-\mathbf{u}^{(j)}\right|_{\infty} .
\end{aligned}
$$


But $X \in \Lambda$ so there exist infinitely many $\mathbf{q} \in \mathbb{Z}^{m}$ such that

$$
\left|\mathbf{q} \cdot \mathbf{x}^{(j)}\right|<m|\mathbf{q}| \psi(|\mathbf{q}|)=|\mathbf{q}|^{-\tau}
$$

by choice of $\psi$. Thus $X \in \tilde{W}(m, n ; \tau)$. Hence

$$
\operatorname{dim} \tilde{W}(m, n ; \tau) \geqslant \operatorname{dim} \Lambda \text {. }
$$

It therefore suffices to find a lower bound for $\operatorname{dim} \Lambda$.

LEMMA 2. The family $\mathscr{R}$ is ubiquitous with respect to the function $\rho: \mathbf{N} \rightarrow \mathbb{R}$ where $\rho(N)=m N^{-m / n} \log N$.

The proof relies on the following familiar variation of the Dirichlet box argument.

LEMMA 3. For each $X \in I^{m n}$ and $N \geqslant 2$ there exists a non-zero integer vector $\mathbf{q}$ in $\mathbb{Z}^{m}$ with $|\mathbf{q}| \leqslant N$ such that

$$
|\mathbf{q} X|<m N^{-(m / n)+1} .
$$

Proof. Consider the vectors $q \in \mathbb{Z}^{m}$ with non-negative components such that $|\mathbf{q}| \leqslant N$. There are $(N+1)^{m}$ such $\mathbf{q}$. This gives $(N+1)^{m}$ values of $\mathbf{q} \cdot \mathbf{x}^{(i)}$ and $(N+1)^{m}$ vectors, $\mathbf{q} X$. Since $X$ is in $I^{m n}$ and $|\mathbf{q}| \leqslant N$

$$
|\mathbf{q} X| \leqslant \frac{1}{2} m N \text {. }
$$

Divide the cube with centre 0 and sidelength $m N$ in $\mathbb{R}^{n}$ into $N^{m}$ smaller cubes of volume $m^{n} N^{n-m}$ and sidelength $m N^{1-(m / n)}$. Since $N^{m}$ is less than $(N+1)^{m}$ there are at least two values $\mathbf{q}_{1} X, \mathbf{q}_{2} X$, say, in one small cube. Thus

$$
\left|\mathbf{q}_{1} X-\mathbf{q}_{2} X\right|=\left|\left(\mathbf{q}_{1}-\mathbf{q}_{2}\right) X\right|<m N^{1-(m / n)} .
$$

Taking $\mathbf{q}_{1}-\mathbf{q}_{2}=\mathbf{q} \in \mathbf{Z}^{m}$, so that $|\mathbf{q}|=\left|\mathbf{q}_{1}-\mathbf{q}_{2}\right| \leqslant N$, the lemma follows.

Proof of Lemma 2. In the definitions of the following sets the q's refer to those integer vectors satisfying $|\mathbf{q}| \leqslant N$ and $|\mathbf{q} X|<m N^{1-(m / n)}$ that exist by Lemma 3. Let

$$
E(N)=\left\{X \in \Omega:|\mathbf{q}|<\frac{N}{\log N}\right\}
$$

and

$$
D(N)=\left\{X \in \Omega: \operatorname{dist}\left(X, \partial I^{m n}\right) \geqslant N^{-1}\right\} \backslash E(N) .
$$

Now,

$$
E(N) \subseteq \bigcup_{1 \leqslant r \leqslant N / \log N} \bigcup_{|\mathbf{q}|=r}\left\{X \in \Omega:|\mathbf{q} X|<m N^{-(m / n)+1}\right\}
$$


whence

$$
\begin{aligned}
|E(N)| & \leqslant \sum_{1 \leqslant r \leqslant N / \log N} \sum_{|\mathbf{q}|=r} \frac{m^{n} N^{-m+n}}{|\mathbf{q}|_{2}^{n}} \\
& \ll N^{-m+n} \sum_{1 \leqslant r \leqslant N / \log N} r^{m-n-1} \\
& \ll N^{-m+n} \frac{N}{\log N} \frac{N^{m-n-1}}{(\log N)^{m-n-1}} \\
& =(\log N)^{-m+n} .
\end{aligned}
$$

Therefore, since $m>n, \lim _{N \rightarrow \infty}|E(N)|=0$ and $\lim _{N \rightarrow \infty}|\Omega \backslash D(N)|=0$. Now let $X \in D(N)$ and $\tilde{\mathbf{q}}$ be such that $|\tilde{\mathbf{q}}| \leqslant N$ and $|\tilde{\mathbf{q}} X|<m N^{-(m / n)+1}$ by Lemma 3 . Since $X \notin E(N)$,

$$
\frac{N}{\log N} \leqslant|\tilde{\mathbf{q}}| \leqslant N .
$$

By definition, $|\tilde{\mathbf{q}}|=\left|\tilde{q}_{i}\right|$ for some $1 \leqslant i \leqslant m$. Let $\delta_{j}=-\tilde{\mathbf{q}} \cdot \mathbf{x}^{(j)} /\left|\tilde{q}_{i}\right|, j=1, \ldots, n$ so that $\tilde{\mathbf{q}} \cdot\left(\mathbf{x}^{(j)}+\delta_{j} \mathbf{e}^{i}\right)=0$, where $\mathrm{e}^{(i)}$ denotes the $i$ 'th basis vector. Also,

$$
\left|\delta_{j}\right| \leqslant \frac{m N^{-(m / n)+1}}{|\tilde{\boldsymbol{q}}|} \leqslant m N^{-m / n} \log N .
$$

Therefore $U=\left(\mathbf{x}^{(1)}+\delta_{1} \mathbf{e}^{i}, \ldots, \mathbf{x}^{(n)}+\delta_{n} \mathbf{e}^{i}\right)$ is a point in $\Pi(\tilde{\mathbf{q}})$, and $|X-U| \leqslant m N^{-(m / n)} \log N$. Now, let $\rho(N)=m N^{-(m / n)} \log N$. Then

$$
D(N) \subseteq A(N)=\bigcup_{|\mathbf{q}|<N} B(\Pi(\mathbf{q}) ; \rho(N))
$$

so that $\lim _{N \rightarrow \infty}|\Omega \backslash A(N)|=0$. Thus $\mathscr{R}$ is ubiquitous with respect to the function $\rho$.

By Theorem 1 in [4], we know the following.

Suppose that $\mathscr{R}$ is a ubiquitous system with respect to $\rho$ and that $\psi: \mathbb{R}^{+} \rightarrow \mathbb{R}^{+}$ is a decreasing function. Then

$$
\operatorname{dim} \Lambda \geqslant t,
$$

where $t=\operatorname{dim} \mathscr{R}+\gamma \operatorname{codim} \mathscr{R}$, and

$$
\gamma=\min \left\{1, \limsup _{N \rightarrow \infty} \frac{\log \rho(N)}{\log \psi(N)}\right\} .
$$

In this case $\operatorname{dim} \Pi(\mathbf{q})=(m-1) n, \operatorname{codim} \Pi(\mathbf{q})=n, \rho(N)=m N^{-(m / n)} \log N$ and $\psi(N)=m^{-1} N^{-(\tau+1)}$. Therefore $\gamma=m /(n(\tau+1))$, and so

$$
\operatorname{dim} \hat{W}(m, n ; \tau) \geqslant \operatorname{dim} \Lambda \geqslant(m-1) n+\frac{m}{\tau+1}
$$

for $m>n$.

Now it only remains to find a lower bound when $m \leqslant n$. Firstly the case $m=n$ is dealt with.

To complete the argument giving the lower bound for the Hausdorff dimension the following two lemmas will be needed. 
Lemma 4. For any real interval, $(a, b)$, and set $X \subseteq \mathbb{R}^{k}$, the Hausdorff dimension of the set $\left(X \times(a, b)^{p}\right)$, where $p$ is a positive integer, is

$$
\operatorname{dim}\left(X \times(a, b)^{p}\right)=\operatorname{dim} X+p .
$$

Proofs for $p=1$ can be found in [1] and [9]. A simple induction argument gives the general case.

LEMMA 5. If there exists an onto function $f: X \rightarrow Y$, such that $f$ is one-one and obeys a bi-Lipschitz condition then

$$
\operatorname{dim} Y=\operatorname{dim} X .
$$

This follows from Theorem 1.8, page 10 in [6].

It is readily verified that the set $A \subseteq I^{m m}$ consisting of points of the form

$$
\left(\mathbf{x}^{(1)}, \ldots, \mathbf{x}^{(m-1)}, \sum_{j=1}^{m-1} a_{j} \mathbf{x}^{(j)}\right)
$$

where

$$
\left(\mathbf{x}^{(1)}, \ldots, \mathbf{x}^{(m-1)}\right) \in \tilde{W}(m, m-1 ; \tau)
$$

and

$$
a_{j} \in\left(-\frac{1}{2(m-1)}, \frac{1}{2(m-1)}\right), \quad j=1, \ldots, m-1,
$$

is a subset of $\hat{W}(m, m ; \tau)$. Define the function

$$
f: \tilde{W}(m, m-1 ; \tau) \times\left(-\frac{1}{2(m-1)}, \frac{1}{2(m-1)}\right)^{m-1} \rightarrow A
$$

by

$$
f\left(\mathbf{x}^{(1)}, \ldots, \mathbf{x}^{(m-1)}, a_{1}, \ldots, a_{m-1}\right)=\left(\mathbf{x}^{(1)}, \ldots, \mathbf{x}^{(m-1)}, \sum_{j=1}^{m-1} a_{j} \mathbf{x}^{(j)}\right) .
$$

The linear independence of the vectors $\mathbf{x}^{(j)}, j=1, \ldots, m-1$ ensures that this onto function $f$ is well defined and one-to-one. Now, the linear independence of the $\mathbf{x}^{(j)}$ implies that the Jacobian of $f$ is of maximal rank. The function $f$ is therefore (see [8], Chapter 1) an embedding and thus the range of $f$ is diffeomorphic to its image $A$. This in turn implies that $f$ is locally bi-Lipschitz. This can also be shown using the Rank Theorem, see [7]. So from Lemma 5

$$
\operatorname{dim} A=\operatorname{dim}\left(\tilde{W}(m, m-1 ; \tau) \times\left(-\frac{1}{2}(m-1)^{-1}, \frac{1}{2}(m-1)^{-1}\right)^{m-1}\right),
$$

and from Lemma 4

$$
\begin{aligned}
\operatorname{dim} & \left(\tilde{W}(m, m-1 ; \tau) \times\left(-\frac{1}{2}(m-1)^{-1}, \frac{1}{2}(m-1)^{-1}\right)^{m-1}\right) \\
& =\operatorname{dim} \tilde{W}(m, m-1 ; \tau)+m-1 .
\end{aligned}
$$

Therefore

$$
\operatorname{dim} \tilde{W}(m, m ; \tau) \geqslant \operatorname{dim} A=(m-1) m+\frac{m}{\tau+1} .
$$


The result extends naturally to the set $\tilde{W}(m, n ; \tau)$ with $m \leqslant n$ as follows. Let $A^{\prime}$ be the set of points

$$
\left(\mathbf{x}^{(1)}, \ldots, \mathbf{x}^{(m-1)}, \sum_{j=1}^{m-1} a_{j}^{(1)} \mathbf{x}^{(j)}, \ldots, \sum_{j=1}^{m-1} a_{j}^{(n-m+1)} \mathbf{x}^{(j)}\right),
$$

where $\left(\mathbf{x}^{(1)}, \ldots, \mathbf{x}^{(m-1)}\right) \in \tilde{W}(m, m-1)$ and

$$
a_{j}^{(i)} \in\left(-\frac{1}{2}(m-1)^{-1}, \frac{1}{2}(m-1)^{-1}\right), \quad 1 \leqslant i \leqslant n-m+1 .
$$

As before it is readily verified that $A^{\prime} \subseteq \hat{W}(m, n ; \tau)$. Then take the function

$$
g: \tilde{W}(m, m-1) \times\left(-\frac{1}{2}(m-1)^{-1}, \frac{1}{2}(m-1)^{-1}\right)^{(n-m+1)(m-1)} \rightarrow A^{\prime}
$$

defined by

$$
\begin{aligned}
& g\left(\mathbf{x}^{(1)}, \ldots, \mathbf{x}^{(m-1)}, a_{1}^{(1)}, \ldots, a_{m-1}^{(1)}, \ldots, a_{1}^{(n-m+1)}, \ldots, a_{m-1}^{(n-m+1)}\right) \\
& =\left(\mathbf{x}^{(1)}, \ldots, \mathbf{x}^{(m-1)}, \sum_{j=1}^{m-1} a_{j}^{(1)} \mathbf{x}^{(j)}, \ldots, \sum_{j=1}^{m-1} a_{j}^{(n-m+1)} \mathbf{x}^{(j)}\right) .
\end{aligned}
$$

This function can also be shown to be one-to-one, onto and bi-Lipschitz but the details are omitted. Hence

$$
\begin{aligned}
\operatorname{dim} A^{\prime} & =\operatorname{dim} \tilde{W}(m, m-1) \times\left(-\frac{1}{2}(m-1)^{-1}, \frac{1}{2}(m-1)^{-1}\right)^{(n-m+1)(m-1)} \\
& =(m-1)^{2}+(m /(\tau+1))+(n-m+1)(m-1)=(m-1) n+(m /(\tau+1)) .
\end{aligned}
$$

Therefore for all positive integers $m, n m>1$,

$$
\operatorname{dim} \hat{W}(m, n ; \tau) \geqslant(m-1) n+(m /(\tau+1)),
$$

and from Lemma 1 ,

$$
\operatorname{dim} \hat{W}(m, n ; \tau)=(m-1) n+(m /(\tau+1)) .
$$

If this method is used when $m>n$ the lower bound obtained is smaller than that obtained using ubiquity. It gives the correct lower bound when $m \leqslant n$ because in this case the vectors of $X$ are linearly dependent.

The second part of the theorem again uses Lemma 3. Consider the set

$$
\left\{Y \in \frac{1}{m} I^{m n}:|\mathbf{q} Y|<|\mathbf{q}|^{-\tau} \text { for infinitely many } \mathbf{q} \in \mathbb{Z}^{m}\right\} \text {. }
$$

This set is contained in $m^{-1} I^{m n}$. By Lemma 3, for each $Y=m^{-1} X \in m^{-1} I^{m n}$ there exists a $q$ such that

$$
|\mathbf{q} Y|=\left|\mathbf{q}\left(m^{-1} X\right)\right|=m^{-1}|\mathbf{q} X|<N^{-(m / n)+1} .
$$

In fact there are infinitely many such $\mathbf{q}$ since Lemma 3 holds for all $N \geqslant 2$. If $|\mathbf{q} Y|=\mathbf{0}$ then $\left|k_{\mathbf{q}} Y\right|=0$ for integer multiples $k \mathbf{q}$ of $\mathbf{q}$.

Suppose $\mathbf{q} Y=\mathbf{c} \neq \mathbf{0}$ for all $\mathbf{q} \in \mathbb{Z}^{m}$. For each $N=2,3, \ldots$, there exists $\mathbf{q}=\mathbf{q}(N)$ such that

$$
|\mathbf{q} Y|<N^{1-(m / n)}
$$


Suppose there are only finitely many such $\mathbf{q}$ and let $\tilde{\mathbf{q}}$ be one such. Then the inequality

$$
|\tilde{\mathbf{q}} Y|<N^{1-(m / n)}
$$

holds for infinitely many $N$. But $|\tilde{\mathbf{q}} Y|=|\mathbf{c}|>0$ and given $m<n, N^{1-(m / n)}$ can be made arbitrarily small. Thus there is a contradiction.

Since $|\mathbf{q}| \leqslant N$ and $0<\tau \leqslant(m / n)-1$ we therefore get

$$
|\mathbf{q} Y|<N^{-(m / n)+1} \leqslant|\mathbf{q}|^{-(m / n)+1}<|\mathbf{q}|^{-\tau},
$$

for infinitely many q. Thus when $0<\tau \leqslant(m / n)-1$, every $Y \in m^{-1} I^{m n}$ is a member of the set

$$
\left\{Y \in m^{-1} I^{m n}:|\mathbf{q} Y|<|\mathbf{q}|^{-\tau} \text { for infinitely many } \mathbf{q} \in \mathbb{Z}^{m}\right\} .
$$

Therefore

$$
\left\{Y \in m^{-1} I^{m n}:|\mathbf{q} Y|<|\mathbf{q}|^{-\tau} \text { for infinitely many } \mathbf{q} \in \mathbb{Z}^{m}\right\}=m^{-1} I^{m n} .
$$

Since $W(m, n ; \tau) \supseteq m^{-1} I^{m n}$

$$
\operatorname{dim} W(m, n ; \tau) \geqslant m n
$$

and Lemma 1 gives the final result,

$$
\operatorname{dim} W(m, n ; \tau)=m n
$$

Acknowledgements. I thank my supervisor Maurice Dodson for suggesting this problem and for his help and encouragement and Christopher Wood for his help with the differential topology. I am grateful to the referee for pointing out some errors and making some helpful comments. I am also grateful to the SERC for financial support.

\section{References}

1. J. D. Bovey and M. M. Dodson. The fractional dimension of sets whose simultaneous rational approximation have errors with a small product. Bull. London Math. Soc., 10 (1978), $213-218$.

2. J. D. Bovey and M. M. Dodson. The Hausdorff dimension of systems of linear forms. Acta Arith., 10 (1986), pp. 337-358.

3. J. W. S. Cassels. An introduction to Diophantine approximation (Cambridge University Press, 1957).

4. M. M. Dodson, B. P. Rynne and J. A. G. Vickers. Diophantine approximation and a lower bound for Hausdorff dimension. Mathematika, 37 (1990), 59-73.

5. M. M. Dodson and J. A. G. Vickers. Exceptional sets in Kolmogorov-Arnol'd-Moser theory. J. Phys. A: Math. Gen., 19 (1986), 349-374.

6. K. J. Falconer. The geometry of fractal sets (Cambridge University Press, 1985).

7. K. J. Falconer. Classes of sets with large intersection. Mathematika, 32 (1985), 191-205.

8. V. Guillemin and A. Pollack. Differential topology (Prentice Hall International, 1974).

9. D. G. Larman. On Hausdorff measure in finite dimensional compact metric spaces. Proc. London Math. Soc. (3), 17 (1967), 193-206.

Dr. H. Dickinson,

Department of Mathematics,

The University of York,

Heslington,

York. YO1 5DD
11J13: NUMBER THEORY; Diophantine approximation, transcendental number theory; Simultaneous homogeneous approximation, linear forms.

Received on the 17th of October, 1991. 